Relations industrielles

Industrial Relations

\title{
Michel CROZIER : L'entreprise à l'écoute. Paris, Inter Éditions,
} 1990, 218 p.

\section{Dimitri Weiss}

Volume 45, numéro 3, 1990

URI : https://id.erudit.org/iderudit/050624ar

DOI : https://doi.org/10.7202/050624ar

Aller au sommaire du numéro

Éditeur(s)

Département des relations industrielles de l'Université Laval

ISSN

0034-379X (imprimé)

1703-8138 (numérique)

Découvrir la revue

Citer ce compte rendu

Weiss, D. (1990). Compte rendu de [Michel CROZIER : L'entreprise à l'écoute. Paris, Inter Éditions, 1990, 218 p.] Relations industrielles / Industrial Relations, 45(3), 647-649. https://doi.org/10.7202/050624ar

Tous droits réservés (C) Département des relations industrielles de l'Université Laval, 1990
Ce document est protégé par la loi sur le droit d'auteur. L'utilisation des services d'Érudit (y compris la reproduction) est assujettie à sa politique d'utilisation que vous pouvez consulter en ligne.

https://apropos.erudit.org/fr/usagers/politique-dutilisation/ 
The labour movement's potential to force such a policy debate on the national level in North America appears bleak. The US labour movement has declined to the point where it is but a minor player on the American political scene. It is too busy struggling to survive, nevermind leading the fight for a better tomorrow. And in Canada, as Poulin-Simon points out, the lack of full employment, social solidarity and an institutional framework for corporatist decision-making makes arriving at such a consensus on the nature of national labour market flexibility policy all the more difficult. In Canada, the Free Trade Agreement was not a product of social compromise but arose to meet the short-term demands of the business community. To say FTA has been a source of conflict within Canadian society is an understatement of major proportions. This example points to the difficulties in establishing a flexible labour market policy, which is humane and consensual. Unless these difficulties are overcome, the benefits of the post-war accord will continue to erode. The outcome could be the continued development of a «flexible» North American economy which exacerbates current economic inequities, a prospect which may only antagonize social tensions which are already building within Canada and the United States.

Brad PRAGNELL

The University of Lethbridge

L'entreprise à l'écoute, par Michel Crozier, Paris, Inter Éditions, 1990, 218 pp.

La sociologie des organisations est, aujourd'hui, au centre de l'attention des responsables et des observateurs des entreprises, et nul doute que Michel Crozier ait beaucoup fait pour que son statut, son niveau et son rayonnement, tant parmi les chercheurs que parmi les praticiens, deviennent, dans notre pays, ceux auxquels elle doit légitimement correspondre. Et le recentrage des préoccupations de certains congénères de Crozier, que les exigences des années 80 ont rapproché de la vie réelle des collectivités de travail, en est un témoignage.

Ce livre apparaît comme une preuve, et un point de repère, de ce que Wieviorka et Trink appelaient récemment («Le modèle EDF», La Découverte, 1989) «le grand renversement» de la discipline, «l'essor foudroyant» de la reconnaissance du facteur humain, et «le formidable tournant» dans les pratiques des managers, le discours des consultants et les analyses de «certains sociologues». M. Crozier note bien que «le problème est désormais sociologique»: un nouveau mode d'organisation, fondé sur la coopération (qui n'existe pas sans conflit et qui, d'autre part, requiert plutôt un plus d'organisation qu'un moins) et la communication (d'où l'écoute), sous entend un changement de logique qui s'éloigne de la sacrosainte croyance d'une rationalisation accélérée pour mobiliser les capacités individuelles et collectives.

L'entreprise change de peau, notait il y a peu Federico Butera, fameux sociologue des organisations lui aussi, l'interprète italien le plus attentif et, probablement, le plus autorisé d'une nouvelle frontière de la culture d'entreprise, assise sur la centralité de la ressource humaine - «rare», «fondamentale», «structurante», «décisive» dit Crozier, autour de laquelle s'ordonnent toutes les autres, car c'est elle «qui fait la différence». Toutes les autres - matières premières, techniques, capital - pour lesquelles il faut disposer de ressources humaines qui permettent leur mise en oeuvre et, ajoute Crozier, de la capacité de mobilisation des divers partenaires. Avec cette précision à la clé: on ne mobilise et on ne motive pas les gens, 
on leur offre des occasions de le faire eux-mêmes. Seuls les hommes peuvent se motiver, parce qu'eux, et eux seuls, savent quel objet et quelle action sont en mesure de satisfaire un besoin (voir P. Morin, «La fonction ressources humaines», Les Éditions d'Organisation, 1988).

Un des interlocuteurs de Crozier, responsable d'usine de l'EGP, disait que «le plus important n'est pas la technique, mais c'est de faire marcher les hommes ensemble». En effet, et il n'est pas surprenant que RSO, l'institut que dirige Butera, travaillait, dans le cadre d'une recherche lancée par le MIT, sur la vérification de l'hypothèse selon laquelle il n'y aurait aucune relation entre investissements en technologie et survie de l'entreprise. La technologie diminue les coûts et ouvre de nouvelles opportunités stratégiques, mais ne sert à rien s'il n'existe pas une nouvelle capacité de management.

Pour Crozier, la capacité d'innover dans le produit, dans la technique et dans le rapport au client prime sur la capacité de rationaliser acquise aux dépens de la capacité de réponse et d'initiative des hommes. Mais, ajouterait Butera, le processus d'adaptation aux nouvelles cultures d'entreprises est tout autre qu'indolore. Les meilleures écoles de management - et on aurait aimé que Crozier en parle - enregistrent les nouvelles cultures, mais elles continuent d'insister sur les éléments de rationalité typiques de l'ancienne culture. Beaucoup de managers, ajoute-t-il, et sur ce point Crozier n'en disconviendrait pas, sont toujours convaincus que la meilleure voie est celle d'une organisation qui assure un gouvernement hiérarchique et permet des parcours de carrière verticaux. Entre les cultures anciennes et les nouvelles exigences, il existe un conflit, et il n'est pas rare d'entendre les managers parler d'une «crise d'identité».

Les ressources rares ne sont plus les mêmes et appellent un nouveau management. La logique classique laisse la place à la logique nouvelle, qui constitue le passage décisif à une société post-industrielle, et ce passage requiert un investissement intellectuel conséquent (avec le concept clé de l'apprentissage). L'investissement matériel perd graduellement de sa prééminence avec la reconnaissance progressive de l'importance et de la capacité de réalisation d'investissements immatériels. Pierre Caspar et Christine Afriat en ont magistralement parlé (notamment dans leur livre sur «L'investissement intellectuel», Economica, 1988), tandis que Hiroyuki Itami - qu'on aurait eu intérêt à traduire, ne serait-ce qu'à partir de la version américaine de son ouvrage (1987) - proposait dans «Mobiliser les ressources invisibles» (la vraie base de la force concurrentielle de l'entreprise) une relecture des processus de production, de la technologie, du marketing et des ressources humaines dans un contexte stratégique dynamique.

Les biens «invisibles» ne se matérialisent pas en installations, produits, etc. Ils sont intangibles, n'apparaissent pas au bilan de l'entreprise et constituent, pourtant, le fondement de son pouvoir compétitif: savoir technologique, image de l'entreprise, connaissances accumulées sur le marché et sur les consommateurs, la compétence du management, la culture de l'entreprise... Normalement, un directeur de ressources humaines a un rôle dans la gestion de ces «invisibles»: le développement de leur valeur et la continuelle recherche de cohérence avec la stratégie de l'entreprise.

À lire Crozier, qui expose d'un regard admiratif le cas de GSI, où les fonctionnels sont rares et le DRH absent, nul n'est besoin d'un tel personnage. Il semble que si GSI est une collectivité atypique, la fonction ressources humaines existe bel et bien et que le rôle d'expertiseconseil d'un DRH existe également, réparti entre le directeur administratif et un personnage placé à l'extérieur de l'entreprise: le consultant. La fonction existe, le partage effectué, les responsables d'unités étant entièrement investis des différents aspects opérationnels, avec un «DRH» original, partagé entre l'administratif interne et le consultant externe. Cette firme est en phase de croissance. Que se passerait-il si cette entreprise passait de 2300 personnes à 5000 , par exemple? Que ferait-elle d'un à-coup provenant d'une trop forte croissance? Comment asseoir la faisabilité de sa stratégie, qui se décide en comité de direction en l'absence d'un 
DRH? Celle-ci pourrait paraître, en tenant compte du mode de fonctionnement de l'entreprise et de sa population très diplômée, essentieilement cadre, normale. Mais sí demain, il y avait une récession, un «dégraissage» - ou alors un besoin de négocier collectivement? Inutile d'ajouter que de syndicats et de relations du travail on n'en parle pratiquement pas. De par les cas évoqués, des interlocuteurs interrogés et des populations salariées décrites, les relations industrielles se réduisent à une version contemporaine des relations humaines. S'exprimer sur un tel sujet, n'était, d'ailleurs, pas le propos des enquêtes, ni la préoccupation majeure des entreprises visitées.

Les interrogations à propos de ce cas me semblent pouvoir se rapprocher de celles qu'on pourrait formuler à propos de l'entreprise-réseau, qui s'exprime à travers une variété de formes, et de la possibilité d'instaurer des politiques actives à l'égard d'une vaste frange de personnels, appelée le «second circuit», que les dimensions imposées pour cette recension ne me permettent pas de développer.

Pour beaucoup de managers et de nos apprentis-cadres, le livre de Crozier sera une révélation. Pour d'autres, une formalisation claire et utile de l'air du temps. Pour d'autres encore, un inventaire originellement ordonné et expliqué de concepts et de pratiques actuels dans le domaine du management, des plus recherchés aux plus discourus, ainsi que l'indique pertinemment l'auteur ( «La tentation du discours»). Pour d'autres, enfin, il ne s'agirait que d'antiennes et de leitmotive, résultant d'une démarche intéressante mais obéissant à un souci, d'ordre pédagogique vraisemblablement, exagérément itératif.

Sans être exhaustif, l'inventaire est suffisamment riche et talentueusement articulé pour retenir l'attention de tous. Porté par l'enthousiasme devant certains de ses interlocuteurs, dirigeants d'entreprises, Crozier n'est, pourtant, pas dupe. Il dit, justement, par exemple, que «la pensée managériale obéit en effet à des canons absolus et éphémères, dont le renouvellement rapide est tout aussi contraignant que celui des modes intellectuelles ou vestimentaires». Et on ne peut, alors, éviter de se demander si l'auteur ne s'est pas lui-même interrogé sur la durée de vie de son produit...

Cet ouvrage un peu singulier, truffé de notations justes, par exemple sur les contradictions typiques de notre temps ou sur la DPPO, acceptée dans son principe et qui soulève tant de problèmes dans son application, avec des objectifs non négociés, aurait mérité, pour lui rendre justice, un véritable review-article, voire un «forum».

Il est à lire nécessairement, avec le même esprit critique dont Crozier a fait preuve à l'égard de certaines des idées ou des faits observés. Résultant d'un long travail (les premières enquêtes datent de 1984-1985) effectué à la demande de l'Insiitut de l'Entreprise, il doit sa substance, outre aux aptitudes connues et reconnues de son auteur, également à ceux qui ont déblayé une portion non négligeable du terrain de départ, et notamment à Françoise Chevalier.

\section{Dimitri WEISS}

Institut d'administration des entreprises

Université de Paris 1

Panthéon-Sorbonne 